\title{
A Method to Predict Track Geometry-induced Vertical Vehicle Motion
}

\author{
Atsushi FURUKAWA, Dr. Eng. \\ Senior Engineer, \\ Track Geometry and Maintenance G., Track Technology Div. \\ Akiyoshi YOSHIMURA, Dr. Eng. \\ Professor, \\ Computer Science Dept., Tokyo University of Technology
}

\begin{abstract}
In this study, the authors use parametric models to predict the track geometry-induced dynamic motion of a vehicle. The characteristics of vehicle dynamics in these models are directly identified through the spatial as opposed to the frequency domain. One of the former's merits is that we can identify the characteristics of vehicle dynamics with fewer observed signals than when we use spectral analysis, making it easier to obtain signals for identification. Another is that we can determine the parameters to represent dynamic behavior of a vehicle using statistical criteria. With these models, we can predict vertical acceleration of a vehicle and its wheel load as well as estimate track conditions by taking into account both ride comfort and operating safety.
\end{abstract}

Keywords: vertical vehicle dynamic motion, track geometry, ARX-model, state-space model

\section{Introduction}

The amplitude of track geometry waveforms is normally used as an index to estimate the geometrical quality of tracks because it is known that track geometry has a correlation with vehicle dynamic responses, such as acceleration, dynamic wheel load and lateral force. To ensure higher quality track maintenance work, however, it is desirable that the index have a higher correlation with vehicle responses. Therefore, we suggest that the dynamic behavior of the vehicle, predicted by means of measured track geometry, can be useful as an index.

There are several ways to predict a dynamic system. The theoretical method that involves solving kinematic equations is the most popular ${ }^{1)}$, but spectral analysis is also used. However, putting these methods into practice for track maintenance work is beset with problems because of the inherent difficulties in deciding on an accurate mathematical model to use and, for example, in determining the vehicle's stiffness and suspension damper rates. Because these values change over time, the solution to a kinematic equation tends to be different from the measured value when these parameters are inaccurate, and the cost of calculation is high. Another factor is that high-capacity computers are needed for theoretical methods, which is not realistic for practical work carried out at track maintenance depots.

Thus, we applied system identification theory to identify the dynamic characteristics of the vehicle to predict how track geometry affected its vertical vibration and dynamic wheel load. The system identification is one of the stochastic signal processing theories similar to spectral analysis, and we can identify the vehicle dynamic characteristics with fewer observed signals than when we use spectral analysis. The main feature of these models is the simplicity of their predictive calculation, which makes it easy to handle these models at track maintenance depots.

The predicted vehicle behavior has higher correlation with actual responses and will provide a more suitable track geometry index than amplitude.

\section{System identification theory ${ }^{2)}$}

\subsection{Outline of the system identification}

We can approximate most dynamic systems as linear systems. By so doing, we can express the relationship between system input and output signals by using Equation (1).

$$
y(n)=\sum_{k=0}^{\infty} g(k) u(n-k)
$$

where $u(n)$ is the input signal; $y(n)$ is the output signal; and $g(k)$ is the system impulse response value. The following function $G(q)$ is termed the system's transfer function:

$$
G(q)=\sum_{k=1}^{\infty} g(k) q^{-k}
$$

where $q$ is the delay operator that has its function expressed by Equation (3).

$$
q^{-1} u(k)=u(k-1)
$$

The task of system identification is to estimate a mathematical model of a system based on observed input-output signals. In the case of the estimation of a dynamic vehicle model, the input signal is the track geometry and the output signal is the vehicle dynamic response. With this theory, we can estimate a vehicle dynamic model once the input and output signals have been observed. As no vehicle structure information is necessary to estimate the model, it is useful for practical track 
maintenance planning.

There are several ways to describe and estimate a system, and this section gives a brief account of the most important approaches. The identification process involves repeatedly selecting a structure, computing the best model of it, and evaluating its properties to see if they are satisfactory.

\subsection{Non-parametric model}

A system is normally added with unmeasurable noise. Therefore, the relationship between the input and output signals must be modified as follows:

$$
y(n)=G(q) u(k)+v(k)
$$

where $v(k)$ is the additional noise.

The transfer function $G(q)$ can be expressed with $q=e^{i w}$ where $\mathrm{w}$ is the unit cycle. The function $G\left(e^{i w}\right)$ is known as the system's frequency response function.

The impulse response $g(k)$ and the frequency-domain description $G\left(e^{i w}\right)$ are called non-parametric model descriptions because they are not defined in terms of a finite number of parameters. The function $G(\omega)$ can be estimated by spectral analysis as follows:

$$
G(\omega)=\frac{S_{u y}(\omega)}{S_{u u}(\omega)}
$$

where $S_{u u}(\omega)$ is the auto-spectrum of the input signal and $S_{u y}(\omega)$ the cross spectrum between input and output signals.

\subsection{Transfer function model}

Rather than specifying the function $G\left(e^{i w}\right)$ in terms of a function of frequency variable $\omega$, we can describe it as rational functions of $q^{-1}$. For this purpose, the ARX (Auto Regressive eXogenous) model that corresponds to

$$
A(q) y(k)=B(q) u(k)+w(k)
$$

is commonly used. $A(a)$ and $B(q)$ are polynomials in the delay operator $q$.

$$
\begin{aligned}
& A(q)=1+a_{1} q^{-1}+a_{2} q^{-2}+\cdots+a_{n_{a}} q^{-n_{a}} \\
& B(q)=b_{1} q^{-1}+b_{2} q^{-2}+\cdots+b_{n_{b}} q^{-n_{b}}
\end{aligned}
$$

Here, the numbers $n_{a}$ and $n_{b}$ are the orders of the respective polynomials. Thus, Equation (7) consists of a finite number of parameters, or $n_{a}+n_{b}$ in this case. Thus, similar models are termed parametric models, of which the ARX model is a typical example.

In an ARX model, the transfer function is defined as follows:

$$
G(q)=\frac{b_{1} q^{-1}+b_{2} q^{-2}+\cdots+b_{n_{b}} q^{-n_{b}}}{1+a_{1} q^{-1}+a_{2} q^{-2}+\cdots+a_{n_{a}} q^{-n_{b}}}
$$

Then the one step forward prediction of the output $\hat{y}(k)$ is obtained thus:

$$
\hat{y}(k)=B(q) u(k)+[1-A(q)] y(k)
$$

Thus prediction errors can be calculated as follows:

$$
\hat{y}(k)-y(k)=B(q) u(k)-A(q) y(k)
$$

The most common method of parametric identification is to estimate the polynomials $A(q)$ and $B(q)$ by minimizing the loss function $V$ which is defined as follows:

$$
V=\sum_{k=1}^{N}\{\hat{y}(k)-y(k)\}^{2}
$$

When the system is presented with the ARX-model, Equation (11) coincides with the linear least square method.

\subsection{State space model}

A common way of describing linear systems is to use the state-space form written as follows:

$$
\begin{aligned}
& x(k+1)=A x(k)+B u(k)+w(k) \\
& y(k)=C x(k)+D u(k)+v(k)
\end{aligned}
$$

Here the relationship between the input $u(k)$ and the output $y(k)$ is defined by using the state vector $x(k)$. In the transfer function form, Equation (12) corresponds to Equation (2) with Equation (13).

$$
G(q)=C^{T}(q I-A)^{-1} B+D
$$

The state space model is more easily treatable than an ordinal ARX model when the system consists of multiple inputs and outputs. In this paper, we apply this model to the wheel load prediction model.

The state space matrices $A, B, C$ and $D$ in Equation (12) can be estimated with the so-called subspace system identification algorithm ${ }^{3)}$.

\subsection{Selecting the optimum model}

Once some estimated models with different structures have been obtained, it is necessary to compare them and to select the optimum one.

If we have a sufficient amount of data, we should fit the partial data set to parametric models, estimate several models whose structures are different, fit another set of data known as validation data to each model and calculate the loss function (see Equation (11)), or the sum of squared prediction errors. Then we can select the structure that has the smallest loss function. This process is known as cross validation and is a good way to approach the model selection problem.

When the model has to be validated on the same data set from which the model has been estimated, the fit always improves as the flexibility of the model structure increases. We need to compensate for this automatic decrease of loss function, for which there are several approaches. The best-known technique is probably Akaike's Final Prediction Error (FPE) criterion ${ }^{4)}$ and his closely related Information theoretic Criterion (AIC). In this paper, we adopted the FPE criterion, which is formed as follows:

$$
F P E=\frac{V}{N} \frac{1+n / 2 N}{1-n / 2 N}
$$

where $n$ is the total number of estimated parameters and $N$ is the number of observed signals. $V$ is the loss function (Equation (11)) for the model structure in question. 


\section{Vertical acceleration prediction model}

\subsection{Identification of vertical vehicle vibration system}

It is known that linearity exists between the vertical alignment of track geometry and the vertical vibration of rolling stock ${ }^{5}$. Thus the transfer function of vertical vibration can be estimated in the frequency domain through spectral analysis (see Equation (5)). For this, the input and output signals must be assumed to be stationary and have a certain value. However, it is difficult to obtain such signals on the narrow-gauge lines in Japan, because trains often accelerate and decelerate according to the speed restrictions in curved sections. Thus we will apply the parametric models for the identification of the vertical vibration system of vehicles.

As an example, the frequency response function (FRF) estimated with three types of algorithm is shown in Fig. 1.

In Fig. 1, the conditions of calculation were as follows:

Input signal: Average vertical alignment of left and right rails

Output signal: Vertical acceleration measured on the front bogie

Data length: $2000 \mathrm{~m}$

Data sampling interval: $1 \mathrm{~m}$ (2000 readings)

Vehicle type: Shinkansen test vehicle

Velocity: $300 \mathrm{~km} / \mathrm{h}$

Frequency band: $0.01-0.1667[1 / \mathrm{m}](0.8-13[\mathrm{~Hz}]$ in the time frequency domain)

The closed circles in Fig. 1 denote the FRF estimated using the ARX model; the closed squares are derived from the state space model; and the closed triangles were estimates using the non-parametric model. The triangles show irregular readings because of the estimation errors in the frequency domain. Since the circles and tri-
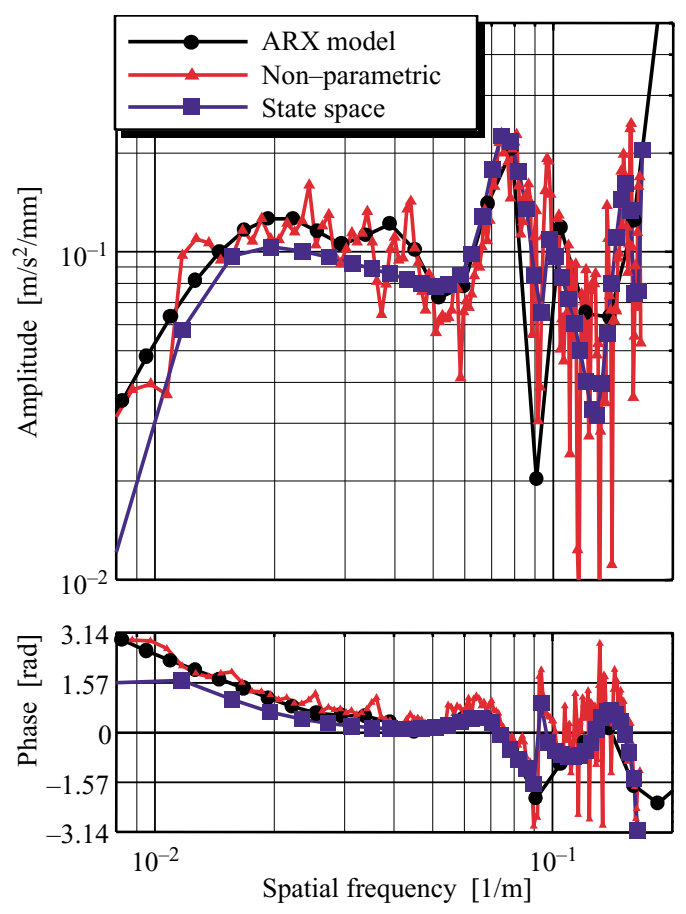

Fig. 1 Frequency Response Function (FRF) of vertical acceleration

angles look smooth, we can expect these lines approximately represent the real system.

Figure 2 shows the predicted and measured vertical accelerations, Fig. 3 the correlation between the predicted and measured waveforms. The similarity between the two waveforms is obvious. Figure 2 also shows the $40 \mathrm{~m}$ chord versine waveform, which is widely used as an index for Shinkansen track geometry. The $40 \mathrm{~m}$ chord versine waveform was calculated from the equation as a function of $10 \mathrm{~m}$ chord versine data similar to the ARX

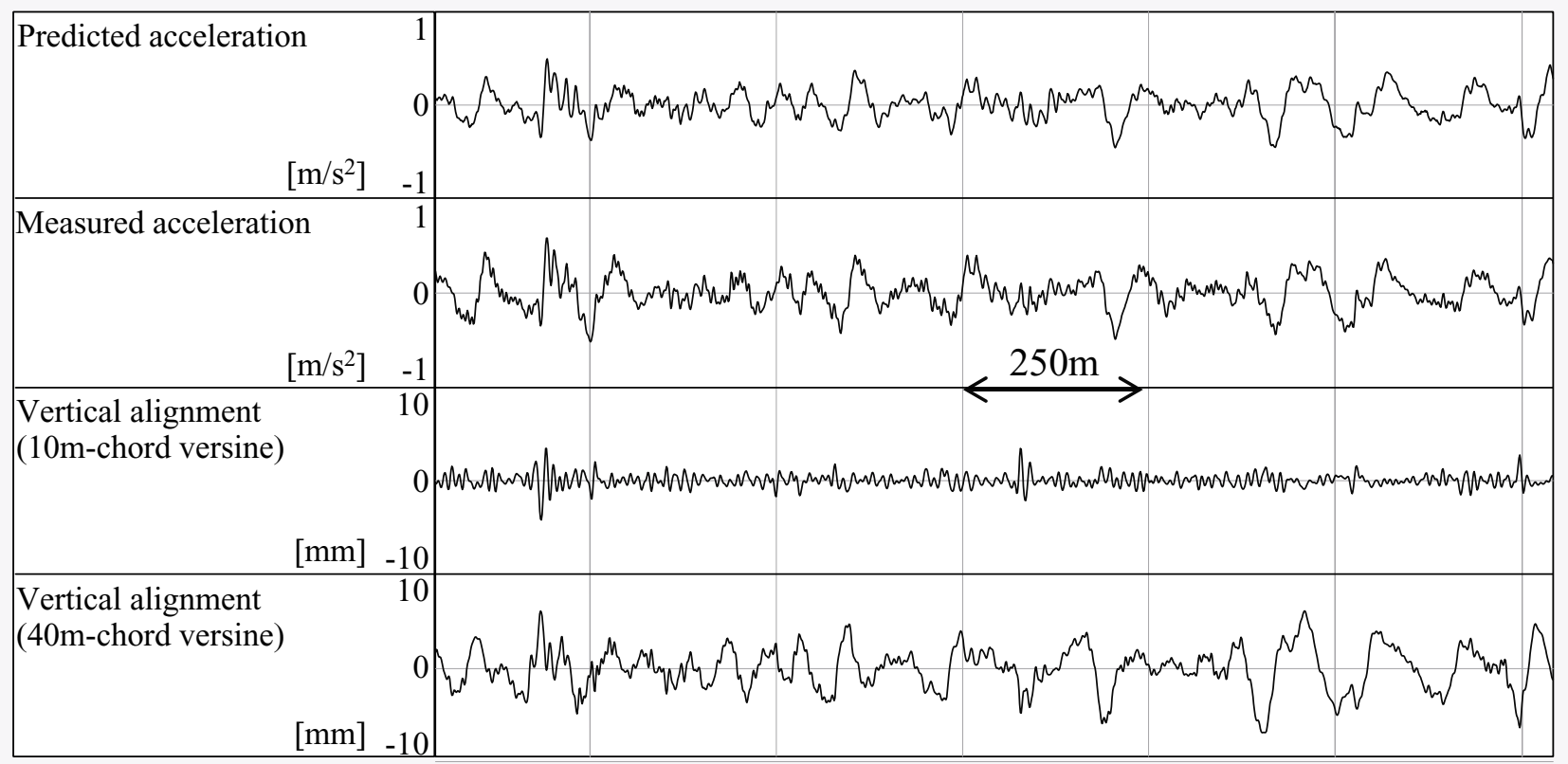

Fig. 2 Predicted and measured vertical acceleration waveforms (Frequency band $=0.01 \sim 0.16[1 / \mathrm{m}]$ ) 


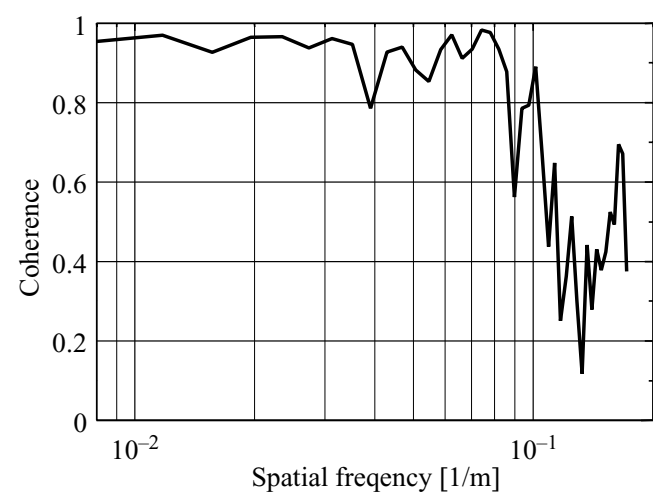

Fig. 3 Correlation between predicted and measured waveforms

model.

The predicted acceleration waveform is more similar to the measured acceleration than the $40 \mathrm{~m}$ chord versine. Hence the acceleration predicted with the ARX model is more suitable for the track geometry index than the traditional versine waveform.

\subsection{Influence of the amount of data}

The main feature of the parametric model is that fewer observed signals are needed than with the nonparametric model, because a system is identified directly with the parametric model in the time/space domain. Next we will confirm the relationship between the accuracy of the estimated model and the data length.

Figure 4 shows the relationship between the Final Prediction Error (Equation (14)) and the order of the models with a different number of signals. In this case, the optimum model, which was the model with the least FPE, is estimated with the least number $(200 \mathrm{~m})$ of signals. The reason is that the slightest variation of train velocity in the section where we recorded the acceleration affected the loss function.

Another topic we should mention about parametric model features is that we can determine the optimum model structure with FPE or other stochastic criteria. In the case of the non-parametric model, we must choose the spectral window and the length of the number response (see Eq. (2)). It is difficult to determine these parameters, however, because there is no criterion on the choice of the optimum one.

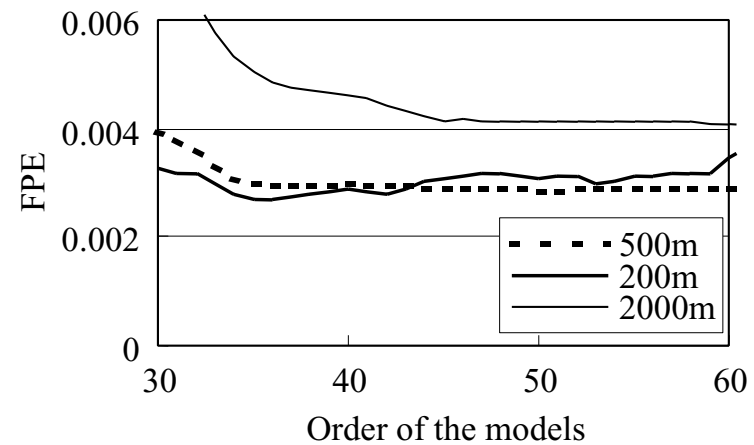

Fig. 4 Final Prediction Error (FPE) in different dataset lengths

\section{Dynamic wheel load prediction model}

\subsection{Relationship between dynamic wheel load and track geometry}

The linearity between the vertical acceleration of a vehicle and track vertical alignment is widely known. On the other hand, the relationship between dynamic wheel load and track geometry is not clear because the dynamic wheel load is affected by various track geometry components, making it difficult to formulize such a system. Therefore, we will consider this relationship before we identify the system.

Figure 5 shows the correlation between dynamic wheel load and track geometry such as the cross level, horizontal alignment and vertical alignment. Frequencies at which the correlation between wheel load and each type of track geometry is high differ from each other.

At frequencies lower than $0.02[1 / \mathrm{m}]$, the wheel load correlates with the horizontal alignment. In this band, the variation of the wheel load is mainly caused by the below-center rolling motion according to the horizontal alignment. At middle frequencies, 0.03 to $0.05[1 / \mathrm{m}]$, the wheel load correlates with the vertical alignment. This band corresponds to the primary natural frequency of a car body in vertical vibration. Thus the vertical vibration of the car body is the main cause of variations in wheel load in this band. At higher frequencies, 0.06 to $0.1[1 / \mathrm{m}]$, the wheel load correlates with the cross level. In this band, the twist of the track, which is the difference of the cross level, affects the dynamic wheel load. The wheel load correlates slightly with the cross level at low frequencies because the cross level affects the belowcenter rolling motion in the same way as horizontal alignment.

As a result, we consider the system as a model of three input signals and one output signal, and identify it with a state space model.

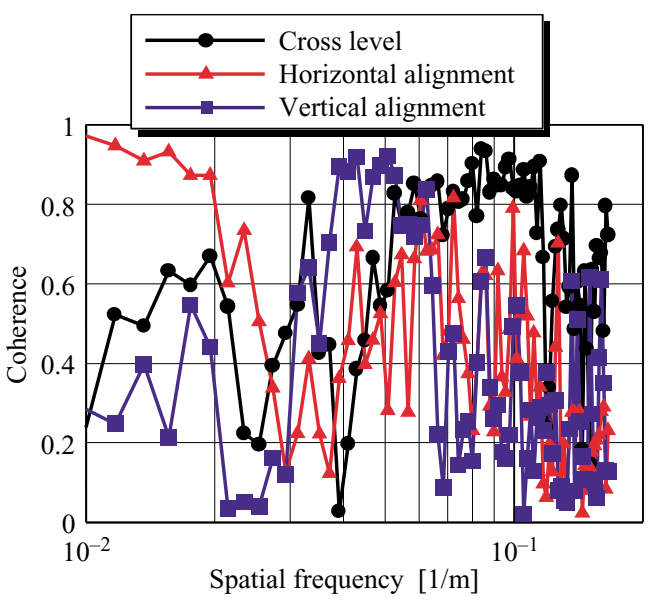

Fig. 5 Correlation between measured wheel load and track geometry

\subsection{Identification of dynamic wheel load}

Figure 6 highlights the similarity between the predicted and measured vertical dynamic wheel load wave- 


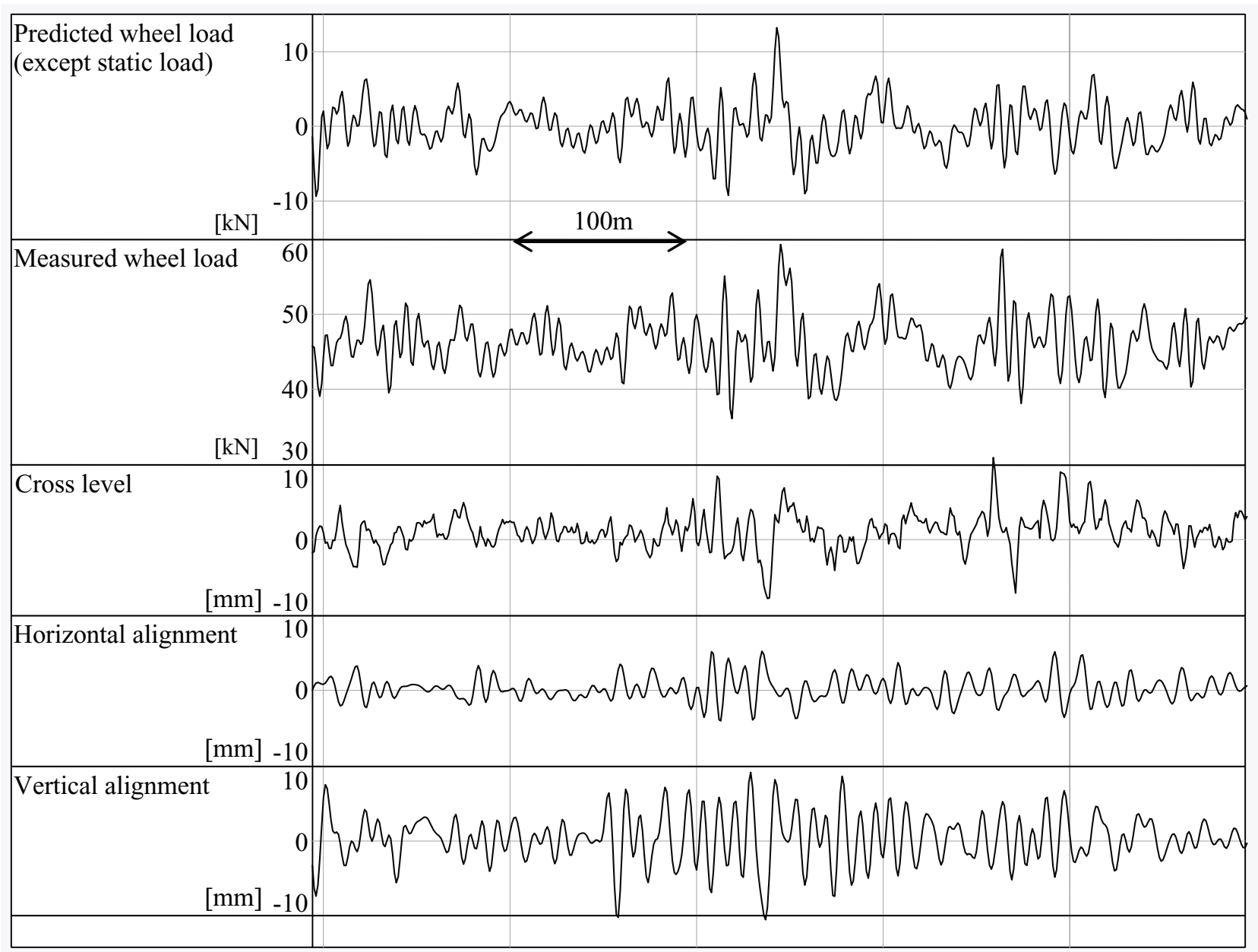

Fig. 6 Predicted and measured vertical dynamic wheel load waveforms (Frequency band $=0.01 \sim 0.16[1 / \mathrm{m}]$ )

forms.

The conditions of calculating Fig. 6 are as follows.

Input signal: Horizontal alignment, vertical alignment and cross level

Output signal: Dynamic wheel load except the static load

Data length: $500 \mathrm{~m}$

Data sampling interval: $1 \mathrm{~m}$

Vehicle type: EMU on some conventional lines

Velocity: $105 \mathrm{~km} / \mathrm{h}$

Frequency band: $0.01-0.1667[1 / \mathrm{m}](0.3-4.9[\mathrm{~Hz}]$

in the time frequency domain)

Figure 7 shows the frequency response functions (FRFs) between wheel load and cross level at different velocities. The amplitude at the primary natural frequency changes according to the velocity, but at the middle frequencies, $0.05-0.15[1 / \mathrm{m}]$, increases according to the frequency, irrespective of the velocity. The reason is that the wheel load variation at different frequencies is caused by the twist of the track within the wheel-base and is thus unaffected by the velocity.

Figure 8 shows the FRFs between wheel load and horizontal alignment at different velocities. The amplitude at all frequencies progresses gradually according to the velocity due to the self-induced rolling motion of

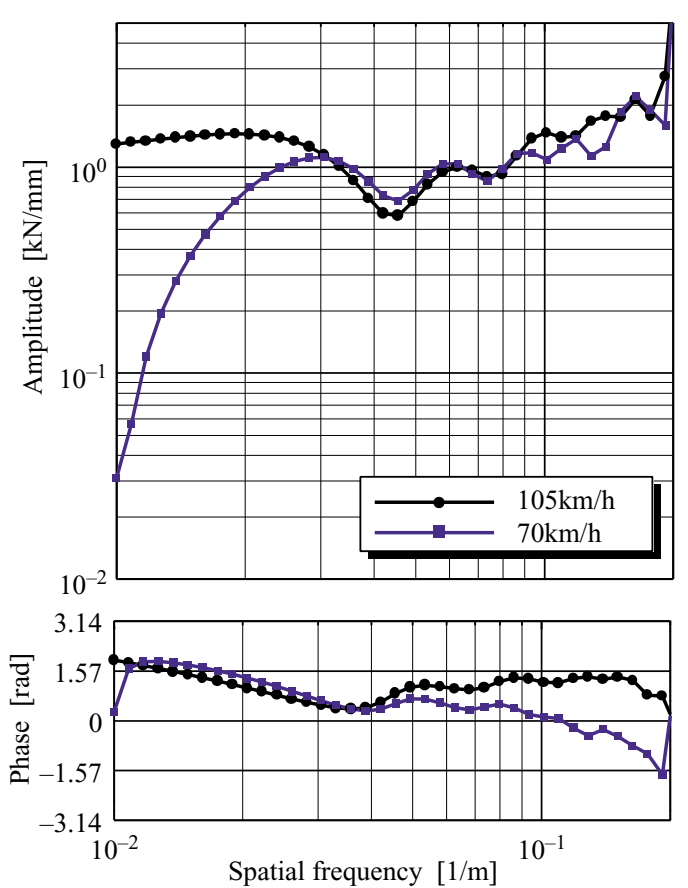

Fig. 7 FRF between wheel load and cross level 

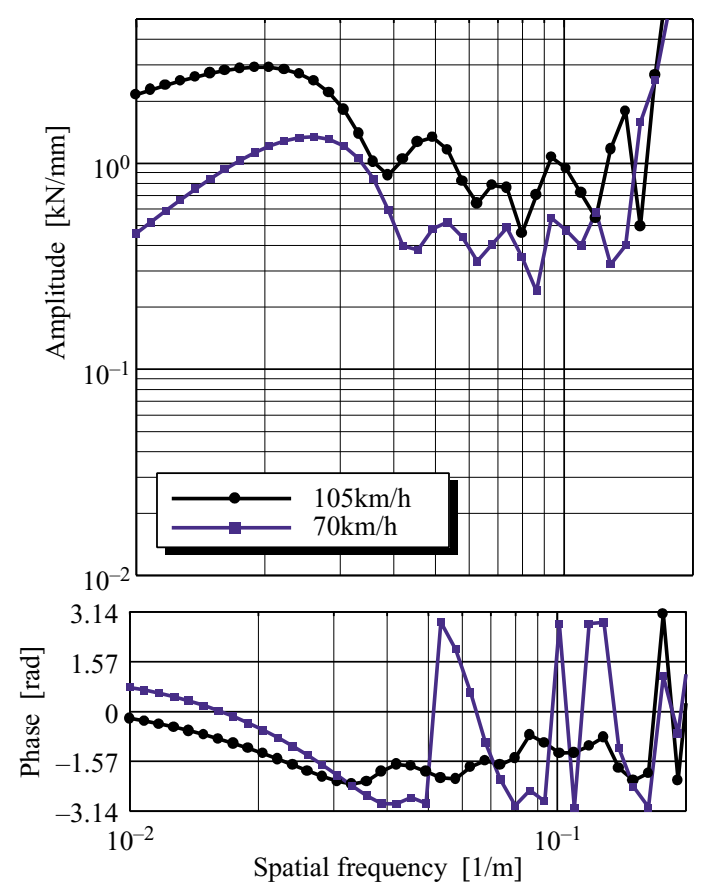

Fig. 8 FRF between wheel load and horizontal alignment
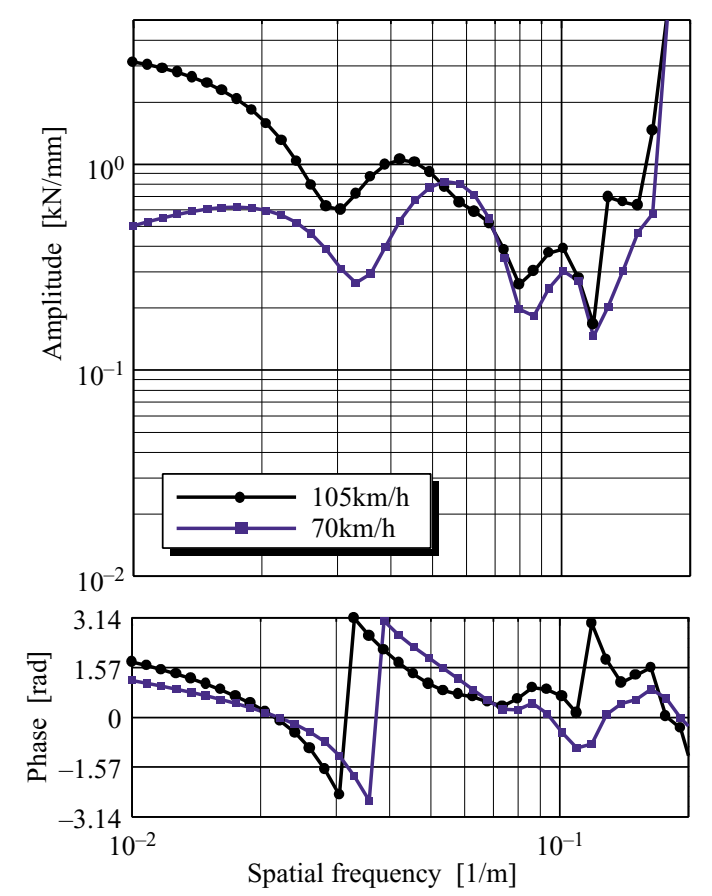

Fig. 9 FRF between wheel load and vertical alignment

the vehicle. The phase differs approximately $\pi[\mathrm{rad}]$ from that in the cross level.

Figure 9 shows the FRFs between wheel load and vertical alignment at different velocities. The primary natural frequency changes according to the velocity. At low frequencies less than $0.03[1 / \mathrm{m}]$ and high frequencies over $0.1[1 / \mathrm{m}]$, wheel loads do not correlate with vertical alignments (see Fig. 5) and so the accuracy of the estimated amplitude at these frequencies is also low. Figure 10 shows the FRFs between vertical acceleration
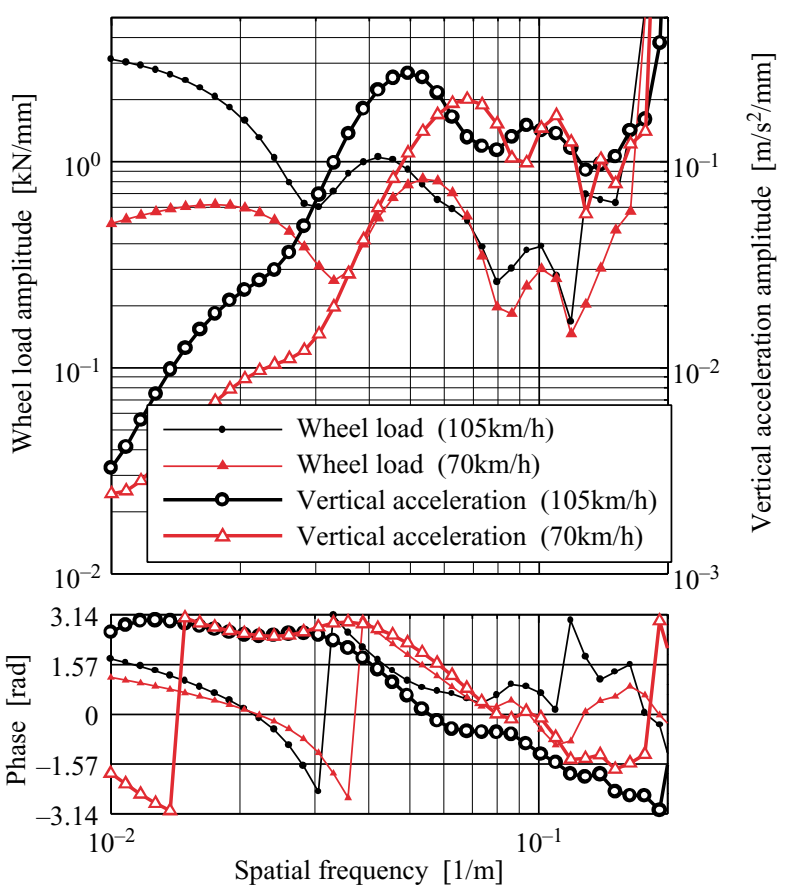

Fig. 10 FRF of vertical vibration and vertical alignment

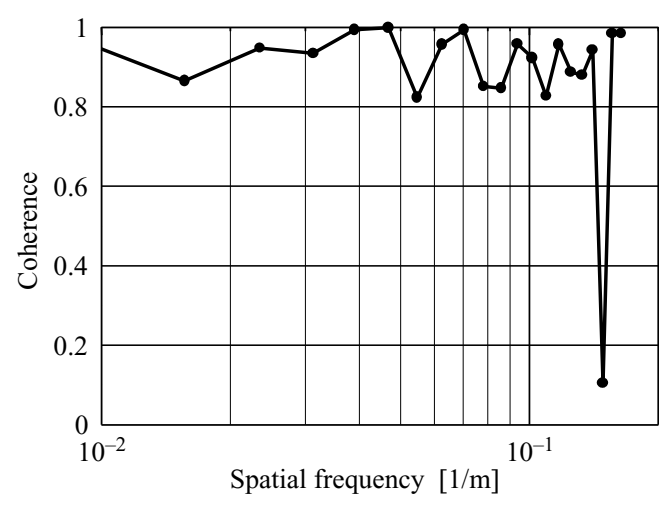

Fig. 11 Correlation between predicted and measured wheel load in circular curve sections

and vertical alignment. The primary natural frequency changes according to the velocity in the same way as the wheel load. Therefore, vertical car body vibration affects the variation of wheel load at the natural frequency.

Figure 11 shows the coherence function between the predicted and measured wheel loads in circular curve sections. We used the parameters estimated from the wheel load measured in a straight section to calculate the predicted wheel load and found that the predicted load correlates with the measured load. Therefore this prediction model is accurate enough for practical applications. We can confirm that the parameters estimated in a straight section are transferable to curved sections.

\section{Conclusions}

The amplitudes of track geometry recorded by the $10 \mathrm{~m}$ chord versine method are used normally as indices 
to estimate track conditions. However, it is known that the waveform measured and recorded by this method is different from its original that represents the true track geometry. Hence it is reasonable to estimate track conditions with an index that indicates the response of rolling stock clearly even if it is different from the true geometry. For this purpose, it is necessary to predict the movement of rolling stock by using the measured track geometry by a simple method.

In this paper we have proposed a stochastic model to predict the vertical acceleration of a vehicle and the wheel load to estimate the track geometry. To develop a model for this purpose, we applied parametric models to identify the dynamic characteristics of the vehicle.

With these models, we can easily estimate the ride quality and operational safety of vehicles without measuring acceleration or wheel loads. We can also plan track maintenance work according to the result of estimation, and estimate the dynamic characteristics of rolling stock at different velocities with these models.

\section{References}

1) ORE C116 rp.2: "A comparison of theoretical and experimental vehicle behavior using a 2 -axled special vehicle," 1972.10.

2) Ljung, L.: "System Identification - Theory For the User, 2nd Edition," PTR Prentice Hall, Upper Saddle River, N.J., 1999.

3) Overschee, P. V. and Moor, B. D.: "Subspace Algorithms for the Identification of Combined Deterministic- Stochastic System," Automatica, Vol.30, No.1, pp.75-93, 1994.

4) Akaike,H.: "Information theory and an extension of the maximum likelihood principle," 2nd, Inter Symposium on Information Theory, Akademiai Kiado, Budapest, 1973.

5) Esvelt, C.: "Modern Railway Track, 2nd Edition," pp.137-151, MRT-Productions, 2001 